\title{
Influencia del gradiente de luz en la fisiología de las bacterias fotosintéticas del azufre. Respuesta ultraestructural.
}

\author{
Isabel Esteve
}

Departamento de Microbiología. Facultad de Ciencias e Instituto de Biología Fundamental. Universidad Autónoma de Barcelona.

\author{
SUMMARY \\ THI: INILLUI:NCE OF LIGHT GRADIENTS ON THE PHYSIOLOGY OF SULFUR \\ PHOTOSINTHETIC BACTERIA. ULTRAESTRUCTURAL RESPONSE
}

Photosyn thetic bacteria are able to maintain their metabolism active in deep waters with very low light intensities using materials accumulated in inclusions during previous photosyn thetic activities. Cisó is a small, anaerobic, sulfur rich pond west of Banyoles lake (Giro$\mathrm{na}$, NE Spain). Due to the massive grow th of the red sulfur photosy nthetic bacterium Chromatium minus, water turns red during several times of the year.

Ultrastructural analysis of cells sampled at different depths in Cisó pond (therefore exposed to different light intensities) showed drastic changes in intracitoplasmic inclusions. Cells which were exposed to $0.01 \%$ surface light intensity in the pond showed a marked increase in poly-B-hidroxybutyrate (PHB) granules and a parallel decrease in sulfur globules, suggesting photosynthetically accumulated glucogen is utilized and converted to PHB with intracellular sulfur as electron acceptor. On the other hand, pure Thiocapsa roseopersicina cultures (another red sulfur bacterium) grown at different, but constant light intensities in the laboratory, never showed changes in their intracytoplasmic inclusions. Instead, under light limiting conditions, they increased the size of their membrane system.

\section{INTRODUCCION}

Las bacterias fotosintéticas precisan para su crecimiento de la luz como fuente de energía y de compuestos reducidos de azufre $\left(\mathrm{H}_{2} \mathrm{~S}, \mathrm{~S}\right.$, tiosulfato) como fuente de poder reductor. No obstante, en los ambientes acuáticos en los que dichas bacterias crecen, la intensidad de la luz decrece exponencialmente al aumentar la profundidad, no llegando por lo general a las capas anóxicas profundas más del 1 al $0,01 \%$ de la luz incidente en superficie. En cultivos puros de laboratorio, las bacterias fotosintéticas se adaptan a condiciones limitantes de luz, mediante modificaciones en su aparato fotosintético (BROCH-DUE \& ORMEROD 1978; GOLECKI \& OELZE 1980), y en el contenido pigmentario (TAKAHASHI et. al. 1972; PIERSON \& CASTENHOLZ, 1974).

Tanto la fotosintesis bacteriana como el metabolismo celular general a bajas inten- 
sidades lumínicas, han sido investigados separadamente por diversos autores, y siempre a partir de cultivos puros (KONDRATIEVA et. al., 1975; GORLENKO \& KUSNETSOV, 1971). A pesar de ello se conoce muy poco acerca de ambos procesos y de las variaciones ultraestructurales resultantes en el habitat natural, donde además de la luz interyienen otros factores como la concentración de nutrientes, la temperatura y otros (MECHLER \& OELZE, 1978; KAISER \& OELZE, 1980).

En la laguna de Cisó se presentan a lo largo de todo el año, densas masas poblacionales de bacterias fotosintéticas que compiten entre si por la luz y el sulfhídrico. El estudio de dicha laguna resulta particularmente interesante, ya que las capas de agua situadas por debajo de $1 \mathrm{~m}$. de profundidad se encuentran privadas de la luz, debido al fuerte sombreado biológico que se produce por el crecimiento bacteriano de las capas superiores (MONTESINOS, 1983).

Las celulas de Chromatium minus, expuestas a estas condiciones presentan im. portantes alteraciones ultraestructurales a nivel de los gránulos de inclusión como gránulos de glucógeno (Glg) y poli-Bhidroxibutirato (PHB), resultado del metabolismo endógeno que presentan dichas células cuando crecen en ambientes po. bremente iluminados.

E1 propósito de nuestra investigación ha sido el estudio ultraestructural de dos especies de Chromatiaceae: Chromatium minus y Thiocapsa roseopersicina (la primera procedente de la laguna de Cisó, y la segunda de un cultivo puro de laboratorio) expuestas durante su crecimiento a diferentes intensidades de luz, con el fin de poder interpretar el metabolismo del que se valen dichas células para mantener su nivel energético en condiciones en que no es posible el crecimiento fotosintético.

\section{MATERIAL Y METODOS}

\section{AREA DE ESTUDIO}

La laguna de Cisó es una pequeña masa de agua situada al oeste del lago de Banyoles
(Girona, Spain). La laguna tiene una morfología rectangular de $457 \mathrm{~m}^{2}$, un volumen de $2.334 \mathrm{~m}^{3}$ y una profundidad maxima de $11 \mathrm{~m}$. (GUERRERO \& ABELLA, 1978). La laguna está rodeada de una vegetación densa que la protege del viento y dificulta la mezcla vertical de la columna de agua.

\section{CULTIVOS CELULARES}

Los cultivos de Thiocapsa roseopersicina se obtuvieron utilizando medio Pfennig (PFENNIG, 1965) con incubación a $25^{\circ}$ y a 1.500 lux.

\section{CARACTERIZACION MORFOLOGICA POR TINCION NEGATIVA}

Se han utilizado básicamente dos colorantes: acetato de uranilo (AU) (WATSON, 1958) y ácido fosfotungstico (PTA) (HALL et al., 1945), ambos en solución acuosa y alcohólica. En el primer caso se empleó AU al $1 \%$ en agua destilada, o en etanol al $70 \%$ y en el segundo PTA al $0,5 \%$ en agua destilada, o en alcohol etilico al $70 \%$.

Para el examen de las muestras por microscopia electrónica de transmisión (TEM), un volumen de $0,01 \mathrm{ml}$ era depositado sobre rejilla de 400 mesh cubierta con película de carbono durante 15 minutos, realizándose a continuación la tinción de la muestra durante dos minutos.

\section{CARACTERIZACION MORFOLOGICA POR MICROSCOPIA ELECTRONICA DE BARRIDO (SEM)}

Para el estudio morfológico bacteriano se hizo pasar un volumen de una muestra concentrada de agua a través de un filtro Nuclepore de 0,2 micras de diámetro de poro, con el fin de retener sobre el filtro los microorganismos de tamaño más pequeño. El filtro se hizo flotar durante 2 horas en glutaraldehido al 2,5\% tamponado con cacodilato o fosfato y a continuación se efectuaron una 


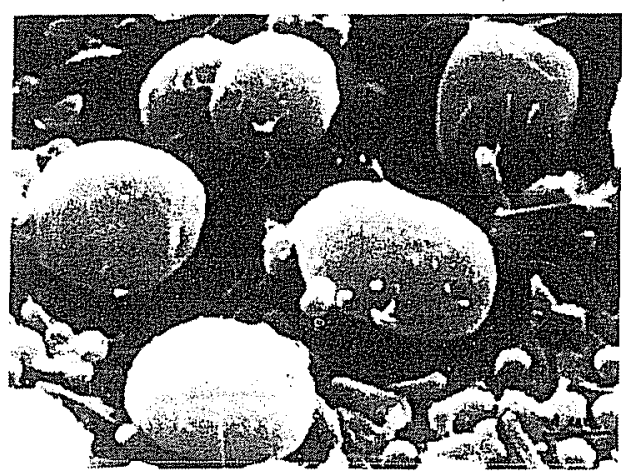

Figura 1. Micrografía electrónica de barrido de células de Chromatium minus (grandes) retenidas sobre filtro Nuclepore.

serie de lavados en tampón sin fijador de 15 minutos de duración, siempre dejando flotar el filtro en la disolución correspondiente. Las deshidrataciones se efectuaron en gradientes de etanol, y finalmente las muestras fueron secadas al punto crítico y sombreadas con oro.

\section{SECCIONES ULTRAFINAS}

Para el análisis ultraestructural se utilizó la técnica de RYTER \& KELLENBERGER (1958). Para ello un sedimento celular obtenido por centrifugación de las muestras á $8.000 \mathrm{~g}$. durante 15 minutos fue fijado con tetróxido de osmio y posteriormente endurecido con agar al $2 \%$, para ser lavado, deshidratado e incluido finalmente en aralditas.

Los cortes ultrafinos fueron teñidos con acetato de uranilo al $1 \%$ en disolución alcohólica al $70 \%$ y con citrato de plomo según la técnica de REYNOLDS (1963).

\section{RESULTADOS}

Las dos especies de bacterias fotosintéticas estudiadas, Chromatium minus y Thiocapsa roseopersicina, pertenecen a la familia

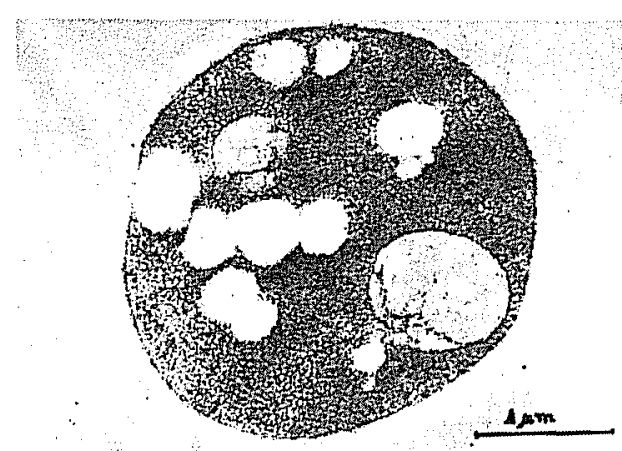

Figura 2. Sección ultrafina de Chromatium minus, mostrando las vesículas fotosintéticas. Las inclusiones de mayor tamaño corresponden a gránulos de PHB (blancos) y glóbulos de azufre (grises).

Chromatiaceae, y presentan como características comunes, una morfología esférica ovoidal (fig. 1) y un sistema intracitoplasmático de membranas de tipo vesicular (fig. 2). Ambas especies se multiplican por escisión binaria y forman limos bajo condiciones especiales. Mientras que la primera es una especie móvil, la segunda es inmóvil y sus células en parejas o tétradas se rodean de una densa cápsula.

Experiencias de campo y de laboratorio efectuadas con Chromatium y Thiocapsa, demuestran que se producen variaciones ultraestructurales importantes cuando dichas bacterias crecen con intensidades limitantes de luz.

Thiocapsa roseopersicina, cuando crece en medio Pfennig a diferentes intensidades de luz $(5,45,1.500$ y 10.000 lux $)$ muestra diferencias en el tamaño de las vesículas fotosintéticas de las células, diferencias que son significativas entre intensidades extremas, con tamaños mayores en células desarrolladas a 5 y 45 lux, y los menores a 10.000 lux. Las figuras 3 y 4 muestran las micrografias electrónicas correspondientes a dichas células cuando se encuentran expuestas a 5 y 10.000 lux respectivamente.

Por otra parte los estudios efectuados en Chromatium minus cuando crece en su habitat natural, prueban que las ma- 


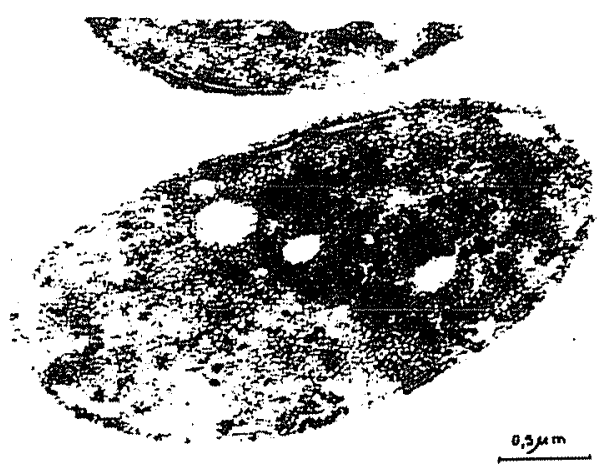

Figura 3. Sección ultrafina de células de Chromatium minus recibiendo una in tensidad lumínica del 1-10\% de la luz incidente en superficie.

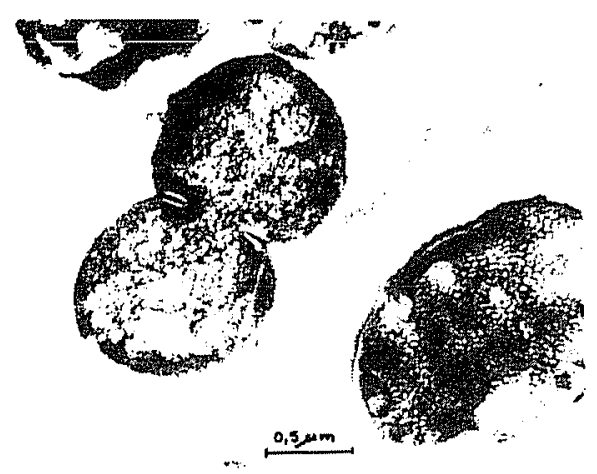

Figura 5. Sección ultrafina de células de Thiocapsa roseopersicina crecidas con una intensidad de luz de 5 lux.

yores diferencias ultraestructurales entre las células desarrolladas a diferentes intensidades de luz radican no tanto en el aparato fotosintético, como en los orgánulos de inclusión; lo que se debe probablemente, a que las células en su ambiente natural presentan movimientos verticales de desplazamiento en la columna de agua que impiden que éstas se encuentren a una intensidad de luz constante, como acontece en experiencias de laboratorio. De esta forma células de Chromatium minus que procedían de la laguna de Cisó al crecer a una intensidad

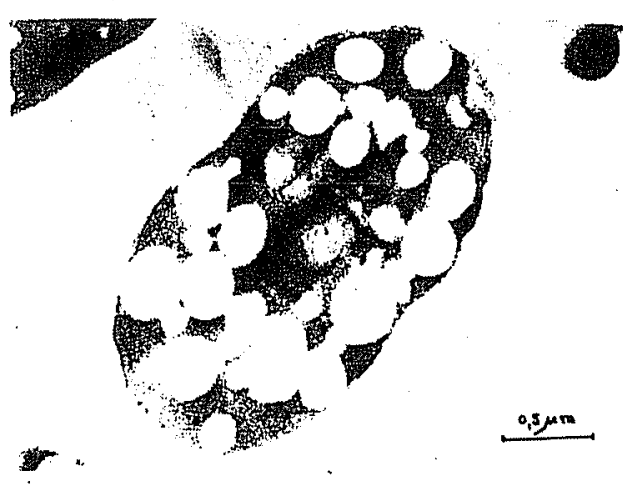

Figura 4. Sección ultrafina de Chromatium minus recibiendo el $0.01 \%$ de la luz incidente en superficie.

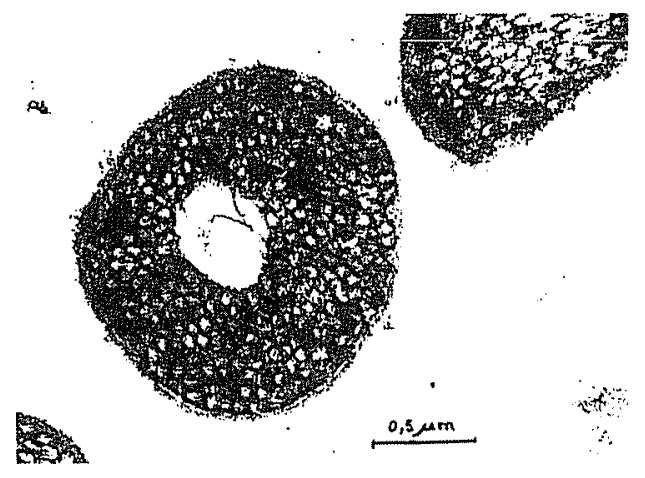

Figura 6. Sección ultrafina de células de Thiocapsa roseopersicina crecidas con una intensidad de luz de 10.000 lux.

luminica entre el $1-10 \%$ de la luz incidente en superficie, muestran los glóbulos de azufre bien conformados y escasas inclusiones de glucógeno y poli-B-hidroxibutirato (fig. 5). Las mismas células al desarrollarse a una intensidad lumínica del $0,01 \%$ de la luz incidente en superficie, muestran los glóbulos degradados y abundantes inclusiones de PHB (ESTEVE et al., 1980) (fig. 6).

Los mismos cambios ultraestructurales han sido observados también en las células de Chromatium procedentes de las lagunas de Estanya (Huesca) y Cullera (Valencia), en 


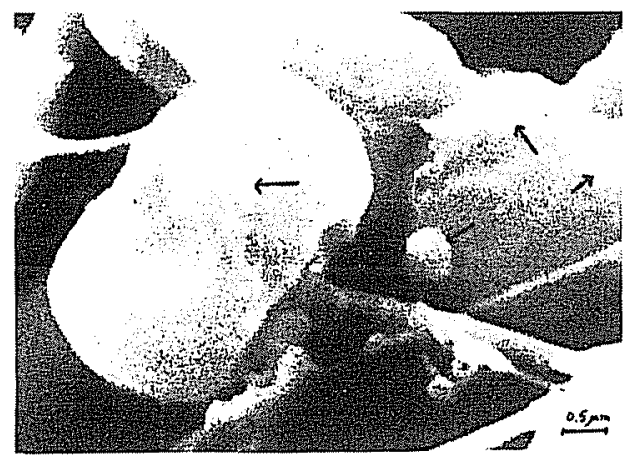

Figura 7. Micrografía electrónica de barrido mostrando celulas epibiontes sobre Chromatium minus (flechas).

las que no se observaron diferencias significativas en el tamaño de las vesiculas fotosintéticas. Tan solo se apreciaron, en este caso, cambios ultraestructurales importantes en las células que crecen en ausencia absoluta de luz, en las que aparecen unas estructuras tubulares en sustitución de las vesiculas fotosintéticas.

Con anterioridad se ha señalado la importancia de las variaciones en la intensidad de la luz en la ultraestructura bacteriana. El estudio de dicho efecto ha servido además para poner de manifiesto la presencia de epibiontes que en ocasiones presentan morfología esférica $(0,6$ micras) y en otras bacilar ligeramente curvada $(0,3 \times 0,6$ micras $)$ y que dependen de la célula huésped Chromatium minus para su reproducción. (figs. 7 y 8).

\section{DISCUSION}

Las bacterias fotosintéticas en sus habjtats naturales estan sujetas diariamente a períodos de carencia de luz. Las carentes de fuente de energía extema dependen de un metabolismo endógeno, es decir de sustancias intracelulares capaces de proporcionar la energía requerida para su propio mantenimiento. El papel que el metabolismo endógeno representa para la supervivencia ha sido ampliamente estudiado (DAWES, 1976;

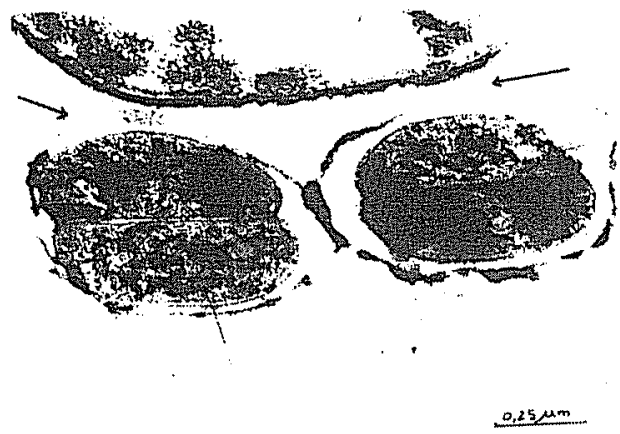

Figura 8. Sección ultrafina de Chromatium minus, presentando varias celulas epibiontes en división.

BREZNAK et al., 1978). Normalmente las reservas celulares, carbohidratos y $\mathrm{PHB}$, son los primeros sustratos para el metabolismo endógeno. Existe entre las bacterias gran variedad en cuanto a su capacidad para resistir el "stress" metabólico, aunque parece existir una correlación entre metabolismo endógeno y supervivencia.

La respuesta fisiológica que experimentan las bacterias fotosintéticas cuando viven privadas de la luz difiere para los distintos grupos. Experimentos efectuados en células de Rhodospirillum rubrum, prueban la capacidad de dichas bacterias de obtener energia a partir de la fermentación de los materiales intracelulares de reserva cuando son transferidas desde unas condiciones de aerobiosis a otras de anaerobiosis en completa oscuridad. Los productos obtenidos de la fermentación del piruvato son: acetato, propionato, formato y $\mathrm{CO}_{2}$ (SHON \& VOELSKOW, 1976).

También las bacterias verdes del azufre (Chlorobiaceae) realizan en la oscuridad un proceso fermentativo. En este caso la poliglucosa acumulada en forma de rosetas internas durante el crecimiento fotosintético, es fermentada en la oscuridad, para dar principalmente: acetato, caproato y succinato (SIREVAG \& ORMEROD, 1977). No obstante los productos obtenidos de la poliglucosa en presencia de luz y en oscuridad son distintos, ya que en la oscuridad la 


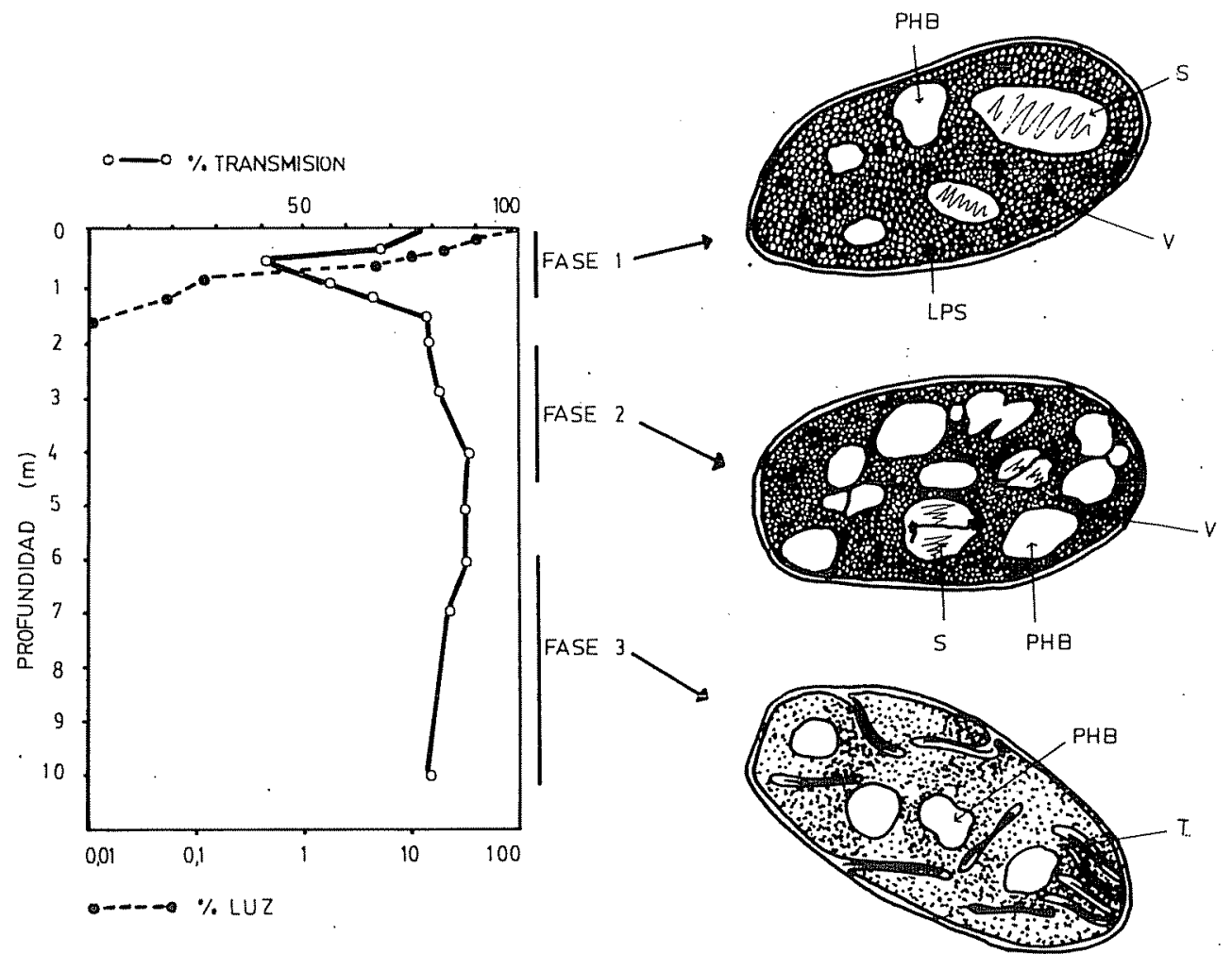

Figura 9. Variaciones en la ultraestructura de Chromatium minus en función de la intensidad de luz que reciben las células cuando crecen en su ambiente natural. Las fases 1 y 2, corresponden a intensidades lumínicas de 0,1 y $0,01 \%$ respectivamente. La fase 3 , corresponde al crecimiento en ausencia de luz. PHB = poli-B-hidroxibutirato. $\mathrm{S}=$ gránulos de azufre. $\mathrm{V}=$ vesículas intracito-plasmáticas. LPS = Glicopolisacárido. $\mathrm{T}=$ túbulos.

poliglucosa sirve como fuente de energía, mientras que en presencia de luz proporciona poder reductor.

La capacidad por parte de las bacterias rojas del azufre (Chromatiaceae) para utilizar reservas endógenas o piruvato anaerobicamente en la oscuridad fue observada por KOHLMILLER \& GEST (1957). No obstante, desde entonces se ha avanzado poco en la respuesta metabólica de este grupo de bacterias.

Los experimentos anteriormente expuestos prueban que cuando las células de Chromatium minus se encuentran creciendo a una intensidad de luz del $0,01 \%$ de la luz incidente en superficie, presentan un incremento muy notable en gránulos de PHB y glóbulos de azufre degradados.

El acúmulo de PHB puede ser interpretado en este caso como resultado del metabolismo endógeno de las células cuando se encuentran en condiciones limitantes de luz o en completa oscuridad. No obstante, el proceso por el que se acumula PHB es aqui distinto al descrito para Rhodospirillum rubrum, por tratarse no de un proceso fermentativo, sino de una respiración endógena en la que el glucógeno acumulado durante el proceso fotosintético es transformado en PHB, mediante la reducción del 
azufre intracelular, que es a su vez convertido en $\mathrm{H}_{2} \mathrm{~S}$. Esta hipótesis estaria de acuerdo con los resultados obtenidos por VAN GEMERDEN (1968) al demostrar que las células de Chromatium vinosum cepa $\mathrm{D}$ desarrolladas en cultivos puros, eran capaces de degradar el glucógeno acumulado en presencia de luz, utilizando el azufre intracelular como aceptor de electrones (VAN GEMERDEN \& BEEFTING, 1978 y BEEFTING \& VAN GEMERDEN, 1979).

Por otra parte células de Thiocapsa roseopersicina, desarrolladas a diferentes intensidades lumínicas no mostraron cambios considerables en las inclusiones citoplasmáticas, debido a que el crecimiento en cultivos puros es rápido, y a que la intensidad de luz fue en cada caso constante, por lo que las células permanecieron poco tiempo expuestas a condiciones limitantes de luz.

La figura 9 representa una interpretación esquemática de los resultados obtenidos a nivel ultraestructural, con el fin de establecer una hipótesis que permita la interpretación fisiológica de los mecanismos de adaptación metabólica de los que se valen las bacterias fotosintéticas del azufre en condiciones limitantes de luz.

BIBLIOGRAFIA

BEEFTING, H.H. \& VAN GEMERDEN, H. 1979. Actual and potential rates of substrate oxidation and product formation in continuous cultures of Chromatium vinosum. Arch. Microbiol, 121: $161-167$

BREZNAK, J.A.; POTRIKUS, C.J.; PFENNIG, N. \& ENSIGN, J.C. 1978. Viability and endogenous substrate used during starvation survival of Rhodospinilum nubium. J. Bacteriol., 134: 381-388.

BROCH-DUE, M. \& ORM EROD, J.G. 1978. Isolation of a Bchl $c$ mutant from Chlorobitum with Bchl $d$ by cultivation at low light intensity. FEMS Microbiology letters, 3: 305-308.

DAWES, E.A. \& SENIOR, P.J. 1973. The role and regulation of energy reserve polymers in microorganisms. Ady. Microb-Physiol., 10: 135-266.

ESTEVE, I.; MONTESINOS, E.; ABELLA, C. \& GUERRERO, R. 1980. Changes in the ultraestructure of the purple sulfur bacteria Chromatium minus depending on the variation of light in a natural habitat. Electron microscopy, 2: 456457 .

GOLECKI, J.R. \& OELZE, J. 1980. Differences in the architecture of cytoplasmic and intracytoplasmic membranes of three chemotrophycally and phototrophically green species of the Rhodospirillaceae. J. Bacteriol., 44: 781-788.

GORLENKO, V.M. \& KUZNETSOV, S.I. 1971. Vertical distribution of Photosynthetic bacteria in lake Kononer Maviys Loy, ASSER. Mikrobiologij'a, 40: 746-752.

GUERRERO, R. \& ABELLA, C. 1978. Dinámica espacio-temporal de las poblaciones bacterianas fotosintéticas en una laguna anaerobia de aguas sulfurosas. Oecol. aquat, 3: 193-206.
HALL, C.E., JAKUS, M.A. \& SCHMITT, F.O 1945. The structure of certain muscle fibrils as revealed by the use of electron stains. $J . A p p l$. Phy's., 16: 459-463.

KAISER, P. \& OELZE, J. 1980. Temperature dependence of membrane-bound enzymes of the metabolism in Rhodospirillum rubrum and Rhadopseudomonas sphaeroides. Arch, Microbiol., 126: 195-200.

KOHLMILLER E.F. \& GEST, H. 1957. A comparative study of the light and dark fermentation of organic acids by $R$ hodospirillum rubrum. $J$ Bacteriol., 61: 269-282.

KONDRATIEVA, E.N.; PETRUSHKOVA, Y.P. \& ZHUKOVA, V.G. 1975. Growth and oxidation of sulfur compounds by Thiocapsa roseopersicina in darkness. Mikrobiologiya, 44: 389-394 (en ruso).

MECHLER, B. \& OELZE, J. 1978. Differentiation of the photosynthetic apparatus of Clwoma. tium vinosum, strain D.I. The influence of growth conditions. Arch. Microbiol., 118: 91 97.

MONTESINOS, L. 1983. Crecimiento y actividad de las bacterias fotosintéticas del azufre en rela ción con gradientes físico-químicos en ecosistemas acuaticos. Actas del Primer Congreso Español de Limnologia.

PFENNIG, N. 1965. Anveicherungs kulturen fur röte und grüne schwefelbacterium. Zentralb. Bakteriol, Parasitenkd. Infektionskr. Hyg. Abt. 1: 179-189.

PIERSON, B.K. \& CASTENHOLZ, R.W. 1974 A phototrophic gliding fllamentous bacterium Chloroflexus aurantiacus gen and s.p. nov. Arch. Microbiol., 100: 5-24. 
REYNOLDS, E.S. 1963. The use of lead citrate at hight $\mathrm{pH}$ as an electronopague strain in electron microscopy. J. Cell. Biol., 17: 208-212.

RYTER, A. \& KELLENBERGER, E. 1958. L'inclusion au polyester pour ultramicrotomie. $J$. Uitrstruct., 2: 200-206.

SCHON, G. \& VOELSKOW, H. 1976. Pyruvate fermentation in Rhodospirillum rubrum after transfer from aerobic to anaerobic conditions in the dark. Arch. Microbiol., 107: 87-92.

SIREVAG, R. \& ORMEROD, J.G. 1977. Synthesis, storage and degradation of polyglucose in Chlorobium thiosulfatophilum. Arch. Microbiol., 111: 239-244.

TAKAHASHI, M., SHIOKAWA, K.\& ICHIMURA,
S. 1972. Photosynthetic characteristics of a purple sulfur bacterium grown under different light intensities. Can. J. Microbiol, 18: 1.8251.828 .

VAN GEMERDEN, H. 1968. On the ATP generation by Chromatium in darkness. 1968. Arch. Mikrobiol., 64: 118-124.

VAN GEMERDEN, H. \& BFIFTING, H.H. 1978. Specific rates of substrate oxidation and product formation in autotrophically growing Chromatium vinosum cultures. Arch. Microbiol., $119: 135-143$.

WATSON, M.L. 1958. Staining of tissue sections for electron microscopy with heavy metals. $J$. Biophy's. Biochem. Cy'tol., 4: 727-729. 